\title{
Projeção em Bases Uniformes Não-Ortogonais ${ }^{1}$
}

\author{
I.S. BONATTI2, C.M. AGULHARI, A. LOPES, P.L.D. PERES, FEEC, UNICAMP,
} Cx.P. 6101, 13081-970 Campinas, SP, Brasil.

Resumo. Um método alternativo para o cálculo dos coeficientes da projeção de um sinal em uma base uniforme não-ortogonal é apresentado neste artigo. O método é decomposto em três etapas: determinação da transformação linear que gera uma base ortogonal e uniforme a partir da base original; representação do sinal na base ortogonal; e convolução dos coeficientes da transformação linear com a representação do sinal na base ortogonal.

Palavras-chave. Ortogonalização, Wavelets, Bases não ortogonais.

\section{Introdução}

Bases adequadas para representar sinais são importantes na transmissão ou extração de informações desses sinais. Se as bases são uniformes, isto é, se podem ser expressas por deslocamentos de funções elementares, apenas as funções elementares precisam ser armazenadas na reconstrução dos sinais.

As operações de deslocamento e escalonamento ortogonais de uma função elementar caracterizam as bases denominadas wavelets. Em particular, a eficiência do sistema wavelet aumenta se a função elementar (conhecida como wavelet mãe) é obtida do conjunto de dados analisados [2], [6], [12].

O cálculo dos coeficientes de representação de um sinal em uma base nãoortogonal é dispendioso, principalmente se a dimensão da base crescer significativamente. Neste artigo, é apresentado um procedimento para efetuar o cálculo simplificado dos coeficientes de projeção que passa pela ortogonalização da base inicial. Para tanto, é apresentado o procedimento denominado Ortogonalização Uniforme para a geração de bases uniformes e ortogonais. O procedimento proposto é similar ao conhecido método de Gram-Schmidt, porém garantindo a uniformidade desejada das bases resultantes.

A apresentação do artigo é organizada conforme descrito a seguir. Na Seção 2, o cálculo dos coeficientes de representação de um sinal qualquer em uma base uniforme não ortogonal é apresentado. Na Seção 3, é apresentado o procedimento de ortogonalização uniforme, que é formulado como uma equação quadrática de matrizes com soluções particulares interessantes. A ortogonalização de Gram-Schmidt é obtida pela fatoração triangular de Cholesky [4] da matriz de correlação da base original. Outra solução, baseada em uma fatoração de Schur [4] da matriz de correlação, é

\footnotetext{
${ }^{1}$ Este trabalho foi apoiado pelas agências CNPq e FAPESP

2ivanil@dt.fee.unicamp.br
} 
apresentada e corresponde à raiz quadrada da inversa da matriz de correlação. Tal ortogonalização produz uma base uniforme quando a raiz quadrada da inversa da matriz de correlação existe para uma matriz de dimensão infinita.

O comportamento assintótico da solução por fatoração de Schur — a dimensão da base original tendendo ao infinito - é analisado na Seção 4. A equivalência assintótica entre a matriz de correlação e uma matriz circulante é estabelecida para matrizes de correlação estritamente diagonais dominantes. Neste caso, é mostrado que a solução da equação de ortogonalização com a matriz de correlação substituída pela matriz circulante é assintoticamente equivalente à solução original, sendo também uma matriz circulante que produz a ortogonalização uniforme desejada. A Seção 5 conclui o artigo.

\section{Projeção Ortogonal}

Considere o conjunto $\mathcal{N}$ de $n$ (ímpar) $^{3}$ funções reais linearmente independentes $f_{k}(t), k \in \mathcal{K}$, chamado de base uniforme, dado por

$$
f_{k}(t)=p(t-k T), \quad T>0, \quad T \in \mathbb{R}, \quad k \in \mathcal{K}=\{0, \pm 1, \ldots, \pm(n-1) / 2\},
$$

com $p(t)$ de norma unitária, isto é,

$$
\left\langle p^{2}(t)\right\rangle=\int_{-\infty}^{+\infty} p^{2}(t) d t=1 \quad, \quad p(t) \in \mathbb{R}, \forall t \in \mathbb{R} .
$$

A projeção de um sinal $x(t)$ na base uniforme suposta não-ortogonal é dada por

$$
x(t)=\sum_{k} \mu_{k} p(t-k T)=\sum_{k} \mu_{k} f_{k}(t)
$$

com

$$
\mu=R^{-1}\langle f(t) x(t)\rangle
$$

sendo $f(t)$ o vetor coluna das funções $f_{k}(t)=p(t-k T), \mu \in \mathbb{R}^{n}$ o vetor coluna de coeficientes e a matriz de correlação temporal $R=\left\langle f(t) f^{\prime}(t)\right\rangle \in \mathbb{R}^{n \times n}$ computada como $R=\left[r_{|k-\ell|}\right]$, com $r_{|k-\ell|}$ dado por

$$
r_{|k-\ell|}=\left\langle f_{k}(t) f_{\ell}(t)\right\rangle=\int_{-\infty}^{+\infty} p(t-k T) p(t-\ell T) d t \quad k, \ell \in \mathcal{K} .
$$

Se as funções $f_{k}(t)$ forem linearmente independentes entre si, a matriz $R$ será, por construção, uma matriz definida positiva e portanto não-singular e a determinação dos coeficientes de projeção $\mu_{k}$ do sinal $x(t)$ na base $f(t)$ implica no cálculo da inversa da matriz de correlação $R$. Se a base fosse ortogonal, o cálculo dos coeficientes de projeção seriam obtidos de maneira desacoplada. Conforme mostrado a seguir, os coeficientes $\mu_{k}$ podem ser obtidos através da convolução dos coeficientes de projeção em uma base ortonormal com os coeficientes da transformação linear que ortonormaliza a base original.

\footnotetext{
${ }^{3} \mathrm{O}$ uso de $n$ ímpar é apenas para facilitar a notação e não representa perda de generalidade.
} 
Considere a transformação linear

$$
q(t)=\sum_{k} \alpha_{k} p(t-k T),
$$

tal que a função elementar $q(t)$ que gera uma base uniforme ortonormal $g_{k}(t), k \in \mathcal{K}$, dada por

$$
g_{k}(t)=q(t-k T), T>0, \quad k \in \mathcal{K}=\{0, \pm 1, \ldots, \pm(n-1) / 2\},
$$

com

$$
\left\langle q^{2}(t)\right\rangle=\int_{-\infty}^{+\infty} q^{2}(t) d t=1, \quad q(t) \in \mathbb{R}, \forall t \in \mathbb{R} .
$$

Os coeficientes de representação $\nu_{k}$ do sinal $x(t)$ na base ortonormal $g_{k}(t)$ são dados por

$$
\nu_{k}=\langle x(t) q(t-k T)\rangle
$$

e a representação de $x(t)$ é dada por

$$
x(t)=\sum_{k} \nu_{k} q(t-k T) .
$$

Substituindo a igualdade (2.5) na equação (2.6), tem-se

$$
x(t)=\sum_{k} \nu_{k} \sum_{\ell} \alpha_{\ell} p(t-(k+\ell) T)=\sum_{\ell} \sum_{k} \nu_{k} \alpha_{\ell-k} p(t-\ell T) .
$$

Comparando as equações (2.3) e (2.7), pode-se concluir que

$$
\mu_{k}=\sum_{\ell} \nu_{\ell} \alpha_{k-\ell}=\nu_{k} * \alpha_{k},
$$

isto é, os coeficientes $\mu_{k}$ podem ser obtidos por convolução de $\nu_{k}$ com $\alpha_{k}$.

A equação (2.8) apresenta uma forma alternativa para determinar os coeficientes de representação $\mu_{k}$ de um sinal $x(t)$ em uma base uniforme não-ortogonal. A seguir é descrito um procedimento para determinar bases ortonormais uniformes.

\section{Ortogonalização}

A função de auto correlação é dada pela equação (2.4) e

$$
\left|r_{k}\right| \leq r_{0}=1, \quad k \in\{1, \ldots, n-1\}
$$

é uma conseqüência da desigualdade de Schwarz.

Note que

$$
\lim _{n \rightarrow+\infty} r_{n-1}=0,
$$

devido à equação (2.2) e se $p(t)$ tem duração finita (suporte compacto), então $r_{k}=0$ para $k$ suficientemente grande. 
Se $r_{k}=0$ para $k \neq 0$, então a base descrita na equação (2.1) é uma base ortogonal. Por exemplo, uma base uniforme ortogonal amplamente conhecida é obtida utilizando $p(t)$ dado por

$$
p(t)=\frac{\sqrt{T}}{\pi t} \sin (\pi t / T)
$$

e a função $p(t)$ conduz ao teorema da amostragem de Shannon, que pode ser visto como uma interpolação de sinais limitados em freqüência [9].

Uma base ortogonal permite um cálculo simples e desacoplado dos coeficientes da projeção do erro mínimo quadrático de uma função no espaço linear [11]. Assim, o principal objetivo deste artigo é ortogonalizar o conjunto $\mathcal{N}$ através de um procedimento linear de ortogonalização.

Para ortogonalizar o conjunto $\mathcal{N}$, define-se um vetor coluna $f$ com dimensão $n$ e composta pelas funções $f_{k}(t)$ e a transformação linear real

$$
g(t)=Q f(t)
$$

na qual $Q \in \mathbb{R}^{n \times n}$ é não singular. Define-se ainda a matriz de correlação $R \in \mathbb{R}^{n \times n}$ como

$$
R=\left\langle f(t) f^{\prime}(t)\right\rangle
$$

sendo $f^{\prime}(t)$ o transposto de $f(t)$. Portanto, os elementos são as funções de correlação dadas por (2.4).

Por construção, $R$ é uma matriz Toeplitz simétrica [7] dado que o $(k, \ell)$ ésimo elemento depende somente do valor de $|k-\ell|$. Assim, tal matriz é constante ao longo das diagonais. Como as funções $f_{k}(t)$ são linearmente independentes, a matriz $R$ é também definida positiva pois para todo $x \neq 0 \in \mathbb{R}^{n}$

$$
x^{\prime}\left\langle f(t) f^{\prime}(t)\right\rangle x=\left\langle\left(f^{\prime}(t) x\right)^{\prime}\left(f^{\prime}(t) x\right)\right\rangle=\left\langle\left(f^{\prime}(t) x\right)^{2}\right\rangle>0 .
$$

A matriz de correlação da base formada pelas funções $\left\{g_{k}(t), k \in \mathcal{K}\right\}$ é dada por

$$
\left\langle g(t) g^{\prime}(t)\right\rangle=\left\langle Q f(t) f^{\prime}(t) Q^{\prime}\right\rangle=Q\left\langle f(t) f^{\prime}(t)\right\rangle Q^{\prime}=Q R Q^{\prime} .
$$

A condição de ortonormalização impõe

$$
\left\langle g_{k}(t) g_{\ell}(t)\right\rangle=\left\{\begin{array}{ll}
1, & k=\ell \\
0, & k \neq \ell
\end{array} \quad \Rightarrow \quad\left\langle g(t) g^{\prime}(t)\right\rangle=\mathbf{I} \Rightarrow Q R Q^{\prime}=\mathbf{I},\right.
$$

que pode ser vista como um sistema de equações com $n^{2}$ incógnitas e $n(n+1) / 2$ equações. Como conseqüência, há diferentes maneiras de gerar uma base ortonormal de um dado conjunto de funções linearmente independentes, uma vez que $n(n-1) / 2$ elementos da matriz $Q$ são arbitrários.

Devido às propriedades da matriz $R$, duas soluções particulares da equação (3.2) podem ser obtidas por técnicas simples de fatoração.

A fatoração de Cholesky aplicada à matriz $R$ produz uma matriz triangular inferior $L$ tal que [8]

$$
R=L L^{\prime}
$$


Então,

$$
Q R Q^{\prime}=(Q L)(Q L)^{\prime}=\mathbf{I}
$$

induzindo

$$
Q=L^{-1}
$$

como uma solução óbvia, produzindo a clássica ortonormalização de Gram-Schmidt [4], [1], dada por

$$
g_{k}(t)=f_{k}(t)-\sum_{\ell=-(n-1) / 2}^{k} \frac{\left\langle f_{k}(t) g_{\ell}(t)\right\rangle}{\left\langle g_{\ell}^{2}(t)\right\rangle} g_{\ell}(t), \quad k \in \mathcal{K} .
$$

Tal procedimento não mantém a uniformidade do conjunto original de funções $f(t)$, dado que o primeiro elemento de $g(t)$ é igual ao primeiro elemento de $f(t)$, o segundo elemento é igual ao erro de projeção do segundo elemento de $f(t)$ sobre o primeiro elemento de $g(t)$, o terceiro é igual ao erro de projeção do terceiro elemento de $f(t)$ sobre os primeiros dois elementos de $g(t)$, e assim por diante.

A fatoração de Schur aplicada à matriz simétrica definida positiva $R$ produz uma matriz ortonormal $V$ e uma matriz diagonal $\Omega$ composta, respectivamente, pelos autovetores e autovalores de $R[8]$, de forma que

$$
R=V \Omega V^{\prime} .
$$

Tal decomposição induz a solução simétrica definida positiva

$$
Q=V \Omega^{-0.5} V^{\prime}=R^{-0.5},
$$

pois

$$
Q R Q^{\prime}=\left(V \Omega^{-0.5} V^{\prime}\right)\left(V \Omega V^{\prime}\right)\left(V \Omega^{-0.5} V^{\prime}\right)=\mathbf{I} .
$$

Note que $\Omega$ e $\Omega^{-0.5}$ são matrizes diagonais com elementos reais positivos uma vez que ambas são matrizes definidas positivas [1]. A matriz diagonal $\Omega$ pode ser escrita como $\Omega=\operatorname{diag}\left\{\rho_{1}, \ldots, \rho_{n}\right\}$, sendo $\rho_{k}$ os autovalores da matriz de correlação $R$.

É mostrado na seqüência que, com o aumento de $n$, a transformação linear $Q$ dada por (3.3) produz uma ortogonalização uniforme, ou seja, quando $n \rightarrow+\infty$ os componentes $g_{k}(t)$ verificam

$$
g_{k}(t)=q(t-k T) \quad ; \quad\left\langle g(t) g^{\prime}(t)\right\rangle=\mathbf{I} \quad, \quad k=0, \pm 1, \pm 2, \ldots
$$

sendo que $q(t)$ é a função elementar obtida do algoritmo de ortogonalização uniforme. Em outras palavras, $\left\{g_{k}(t)\right\}$ é uma base uniforme e ortogonal de dimensão infinita.

\section{Comportamento Assintótico da Decomposição de Schur}

Nesta seção, a demonstração de que a solução $Q=R^{-0.5}$ produz uma ortogonalização uniforme baseia-se na propriedade de que a matriz circulante $C$ é assintoticamente equivalente a $R$, se as seguintes condições suficientes forem assumidas para 
qualquer $n$ :

$$
\begin{gathered}
\left|r_{k}\right| \leq \eta^{k} \quad, \quad k \in\{0,1, \ldots, n-1\}, \\
2 \sum_{k=1}^{(n-1) / 2} \eta^{k}<r_{0} \Rightarrow 2 \sum_{k=1}^{(n-1) / 2}\left|r_{k}\right|<r_{0},
\end{gathered}
$$

$\operatorname{com} \eta>0$ o maior valor que satisfaz as equações (4.1) e (4.2). Para que $\eta$ satisfaça ambas as equações tem-se que $\eta<1 / 3$. A suposição dada pela equação (4.1) restringe o quanto a função de auto correlação decai conforme $k$ aumenta, sendo uma conseqüência de como $p(t)$ evanesce com o aumento de $t$ e de quão perto estão os pulsos vizinhos $p(t-k T)$.

Observe que a matriz de correlação $R$ satisfazendo a equação (4.2) é uma matriz estritamente diagonal dominante [4] e, do teorema dos círculos de Gersgorin [1], [10], os autovalores da matriz $R$ estão contidos na união dos círculos cujos centros são os elementos da diagonal de $R$, e os raios de tais círculos são dados pela soma dos valores absolutos dos elementos de cada linha exceto o elemento da diagonal. Como $r_{0}=1$ e os autovalores de $R$ são números reais, o teorema de Gersgorin pode ser explicitado como

$$
\frac{1-3 \eta}{1-\eta} \leq \rho_{k} \leq \frac{1+\eta}{1-\eta}, k \in\{0,1, \ldots, n-1\} .
$$

A matriz circulante simétrica $C \in \mathbb{R}^{n \times n}$ é baseada na matriz de correlação $R$ e é construída por deslocamentos cíclicos do vetor horizontal $c$ definido por

$$
c_{0}=1, \quad c_{k}=c_{n-k}=r_{k} \text { para } k \in\{1, \ldots,(n-1) / 2\} .
$$

A matriz circulante $C$ é uma matriz Toeplitz cuja primeira linha consiste no vetor $c$, a segunda linha é o vetor $c$ deslocado ciclicamente para a direita de uma posição, a terceira linha é igual à segunda linha deslocada ciclicamente para a direita de uma posição, e assim por diante. Por exemplo, $R$ e $C$ para $n=5$ são dados por

$$
R=\left[\begin{array}{ccccc}
1 & r_{1} & r_{2} & r_{3} & r_{4} \\
r_{1} & 1 & r_{1} & r_{2} & r_{3} \\
r_{2} & r_{1} & 1 & r_{1} & r_{2} \\
r_{3} & r_{2} & r_{1} & 1 & r_{1} \\
r_{4} & r_{3} & r_{2} & r_{1} & 1
\end{array}\right] \quad ; \quad C=\left[\begin{array}{ccccc}
1 & r_{1} & r_{2} & r_{2} & r_{1} \\
r_{1} & 1 & r_{1} & r_{2} & r_{2} \\
r_{2} & r_{1} & 1 & r_{1} & r_{2} \\
r_{2} & r_{2} & r_{1} & 1 & r_{1} \\
r_{1} & r_{2} & r_{2} & r_{1} & 1
\end{array}\right]
$$

Note que com exceção dos cantos superior direito e inferior esquerdo, a matriz $R$ é igual à matriz circulante $C$. De fato, pode ser demonstrado que somente $\left(n^{2}-1\right) / 4$ elementos de $R$ são diferentes dos elementos de $C$.

Como $C$ é uma matriz circulante [8], [13], $C$ pode ser escrito como

$$
C=U \Gamma U^{\prime},
$$

com $\Gamma$ sendo uma matriz diagonal composta pelos $n$ autovalores de $C$, dados por

$$
\gamma_{\ell}=\sum_{k=0}^{n-1} c_{k} \exp \left(-j \ell \frac{2 \pi}{n} k\right) \quad \text { para } \quad \ell \in\{0, \ldots, n-1\},
$$


e $U$ é uma matriz unitária composta pelos autovetores de $C$. Os elementos da matriz $U$ são dados por

$$
u_{\ell k}=\frac{1}{\sqrt{n}} \exp \left(-j \ell \frac{2 \pi}{n} k\right) \quad \text { para } \quad k, \ell \in\{0, \ldots, n-1\} .
$$

Note que $U$ não depende de $C$, e $\gamma_{\ell}$ são os coeficientes da transformada de Fourier discreta da primeira linha da matriz $C$. O vetor $c$ é a transformada inversa de Fourier discreta do vetor $\gamma$, dado por

$$
c_{k}=\frac{1}{n} \sum_{\ell=0}^{n-1} \gamma_{\ell} \exp \left(j k \frac{2 \pi}{n} \ell\right) .
$$

Além disso, como $c^{\prime}=c$, os autovalores $\gamma_{\ell}$ são reais. Como $c_{0}=1$ (por construção), tem-se que

$$
\sum_{\ell=0}^{n-1} \gamma_{\ell}=n
$$

O teorema de Parseval resulta em

$$
\sum_{k=0}^{n-1}\left|c_{k}\right|^{2}=\frac{1}{n} \sum_{\ell=0}^{n-1}\left|\gamma_{\ell}\right|^{2}
$$

Note que, por construção, $C$ é uma matriz estritamente diagonal dominante, e portanto

$$
\frac{1-3 \eta}{1-\eta} \leq \gamma_{k} \leq \frac{1+\eta}{1-\eta}, k \in\{0,1, \ldots, n-1\} .
$$

Lema 4.1. As matrizes $C$ e $R$ são assintoticamente equivalentes.

Demonstração. Como $R$ e $C$ são simétricas e seus autovalores satisfazem a equação (4.3), ambas as normas fortes são finitas, pois

$$
\|R\|=\max _{k} \rho_{k} \leq \frac{1+\eta}{1-\eta} \quad, \quad\|C\|=\max _{k} \gamma_{k} \leq \frac{1+\eta}{1-\eta} .
$$

Como conseqüência das estruturas de $R$ e $C$, tem-se que

$$
n|R-C|^{2}=2 \sum_{k=1}^{(n-1) / 2} k\left(r_{n-k}-r_{k}\right)^{2} \leq 2 \sum_{k=1}^{(n-1) / 2} k\left(r_{n-k}^{2}+2\left|r_{n-k}\right|\left|r_{k}\right|+r_{k}^{2}\right),
$$

e também

$$
n|R-C|^{2} \leq 2 \sum_{k=1}^{n-1} k\left(r_{k}^{2}\right)+4 \sum_{k=1}^{(n-1) / 2} k\left|r_{n-k}\right|\left|r_{k}\right|,
$$

e da condição imposta na equação (4.1) tem-se

$$
n|R-C|^{2} \leq 2 \sum_{k=1}^{n-1} k\left(\eta^{2}\right)^{k}+4 \sum_{k=1}^{(n-1) / 2} k \eta^{n} \leq \frac{2 \eta^{2}}{\left(1-\eta^{2}\right)^{2}}+\eta^{n} \frac{(n+1)(n-1)}{2} .
$$

Para $n$ tendendo ao infinito, a norma fraca da diferença entre $R$ e $C$ tende a zero, pois o lado direito da equação (4.5) é finito. 
A prova do Lema 4.1 é baseada em [5], em que prova-se que uma matriz Toeplitz (com um número finito de elementos não nulos) e sua associada matriz circulante são assintoticamente equivalentes. A diferença aqui são as suposições alternativas dadas pelas condições (4.1) e (4.2).

Para $n$ suficientemente grande, as equações (3.1) e (3.2) são respectivamente substituídas pelas equações

$$
g(t)=\mathcal{Q} f(t)
$$

$\mathrm{e}$

$$
\mathcal{Q} C \mathcal{Q}^{\prime}=\mathbf{I}
$$

uma vez que $R$ e $C$ são assintoticamente equivalentes.

A fatoração apresentada na equação (4.4) induz a solução

$$
\mathcal{Q}=U \Gamma^{-0.5} U^{\prime}=C^{-0.5} .
$$

Note que $\mathcal{Q}$ é uma matriz circulante por construção e, conseqüentemente, os autovalores de $\mathcal{Q}$ são dados por

$$
\beta_{\ell}=\frac{1}{\sqrt{\gamma_{\ell}}} \text { para } \ell \in\{0, \ldots, n-1\} .
$$

A inversa da transformada de Fourier discreta dos autovalores de $\mathcal{Q}$ produz sua primeira linha, dada por

$$
b_{k}=\frac{1}{n} \sum_{\ell=0}^{n-1} \beta_{\ell} \exp \left(j \ell \frac{2 \pi}{n} k\right), \text { para } k \in\{0, \ldots, n-1\} .
$$

Por construção

$$
b_{k}=b_{n-k} \text { para } k \in\{1, \ldots,(n-1) / 2\}
$$

e $b_{k}$ satisfaz, para qualquer $n$,

$$
\sum_{k=0}^{n-1} b_{k}^{2}<+\infty
$$

pois

$$
\left|C^{-0.5}\right| \leq\left\|C^{-0.5}\right\|=\max _{k} \beta_{k}=\max _{k} \frac{1}{\sqrt{\gamma_{k}}}<\left(\frac{1-\eta}{1-3 \eta}\right)^{0.5}<+\infty .
$$

O quadrado da norma fraca de $C^{-0.5}$ pode ser escrito como

$$
\left|C^{-0.5}\right|^{2}=n^{-1}\left(n \sum_{k=0}^{n-1} b_{k}^{2}\right)<\frac{1-\eta}{1-3 \eta}<+\infty .
$$

Como $\mathcal{Q}$ é uma matriz circulante por construção, a transformação $g(t)=C^{-0.5}$ $f(t)$ produz uma ortogonalização uniforme para $n$ suficientemente grande e a função $q(t)$ dada por (3.4) pode ser definida como

$$
q(t)=\lim _{n \rightarrow+\infty} \sum_{k=-(n-1) / 2}^{(n-1) / 2} \alpha_{k} p(t-k T), \quad \alpha_{k}=\alpha_{-k}=b_{k} .
$$




\title{
5 Conclusão
}

A determinação dos coeficientes de projeção de um sinal no espaço linear gerado por uma base uniforme não-ortogonal é proposta neste artigo. Os coeficientes de projeção são determinados pela convolução entre os coeficientes de representação do sinal na base uniforme ortogonal e os coeficientes da transformação linear que ortogonaliza a base original. O algoritmo para a geração de bases uniformes ortogonais, a partir de conjuntos não ortogonais e linearmente independentes de funções uniformes é a principal contribuição desse artigo. Uma importante diferença entre a base obtida pelo algoritmo proposto e a base resultante do método de ortogonalização de Gram-Schmidt é que a primeira é uniforme, característica essencial na aplicação em sistemas wavelet. O algoritmo de Ortogonalização Uniforme apresenta um bom desempenho computacional por se basear na transformada rápida de Fourier.

\begin{abstract}
An alternative method for the calculation of the projection coefficients of a signal on a non-orthogonal uniform basis is presented in this paper. The method is decomposed into three steps: determination of the linear transformation which generates an orthogonal uniform basis from the original basis; representation of the signal on the orthogonal basis; and convolution of the coefficients of the linear transformation with the signal representation on the orthogonal basis.
\end{abstract}

\section{Referências}

[1] R. Bellman, "Introduction to Matrix Analysis", Classics in Applied Mathematics, SIAM, vol. 19, second edition, Academic Press Inc., 1997.

[2] J.O. Chapa, R.M. Rao, Algorithms for designing wavelets to match a specified signal, IEEE Transactions on Signal Processing, 48, No. 12 (2000), 3395-3406.

[3] A.L. Goldberger, L.A.N. Amaral, L. Glass, J.M. Hausdorff, P.Ch. Ivanov, R.G. Mark, J.E. Mietus, G.B. Moody, C.-K. Peng, H.E. Stanley, PhysioBank, PhysioToolkit, and PhysioNet: Components of a new research resource for complex physiologic signals, Circulation, 101, No. 23 (2000), e215-e220.

[4] G.H. Golub, C.F. Van Loan, "Matrix Computations", third edition, John Hopkins Press, New York, 1996.

[5] R.M. Gray, On the asymptotic eigenvalue distribution of toeplitz matrices, IEEE Transactions on Information Theory, 18, No. 6 (1972), 725-730.

[6] A. Gupta, S.D. Joshi, S. Prasad, A new method of estimating wavelet with desired features from a given signal, Signal Processing, 85, No. 1 (2005), 147161.

[7] T. Kailath, "Linear Systems", Prentice Hall, Inc., 1980.

[8] P. Lancaster, M. Tismenetsky, "The Theory of Matrices with Applications", second edition, Academic Press Inc., 1985. 
[9] B.P. Lathi, "Signals Processing \& Linear Systems", Oxford University Press, 1998.

[10] C. Meyer, "Matrix Analysis and Applied Linear Algebra", SIAM, Philadelphia, PA, 2000.

[11] A.V. Oppenheim, A.S. Willsky, S.H. Nawab, "Signals \& Systems", Prentice Hall Signal Processing Series. Prentice Hall, second edition, 1996.

[12] L.K. Shark, C. Yu, Design of matched wavelets based on generalized Mexicanhat function, Signal Processing, 86, No. 7 (2006), 1451-1469.

[13] G. Strang, "Introduction to Applied Mathematics", Wellesley-Cambridge Press, 1986. 